\title{
Weak Deflection angle and Shadow by Tidal Charged Black Hole
}

\author{
Wajiha Javed, ${ }^{1, *}$ Ali Hamza, ${ }^{1, \dagger}$ and Ali Övgün ${ }^{2,3, \ddagger}$ \\ ${ }^{1}$ Division of Science and Technology, University of Education,Township Campus, Lahore-54590, Pakistan \\ ${ }^{2}$ Instituto de Física, Pontificia Universidad Católica de Valparaíso, Casilla 4950, Valparaíso, Chile. \\ ${ }^{3}$ Physics Department, Arts and Sciences Faculty, Eastern Mediterranean University, Famagusta, North Cyprus via Mersin 10, Turkey.
}

(Dated: March 2, 2020)

\begin{abstract}
In this article, we calculate the deflection angle of tidal charged black hole (TCBH) in weak field limits. For doing this, first of all we obtain the Gaussian optical curvature and then apply the GaussBonnet theorem on it and with the help of Gibbons-Werner method, we calculate the light's deflection angle by TCBH. After calculating the deflection angle of light, we check the graphical behavior of TCBH. Moreover, we further find the light's deflection angle in the presence of plasma medium and also check the graphical behavior in the presence of plasma medium. In the next section, we investigate the shadow of TCBH. For calculating the shadow, we first find the photon geodesic around the TCBH and then find its shadow. We also obtain TCBH's shadow in the plasma medium. Moreover, we also find shadow's shapes and discuss the effect of plasma on shadow.
\end{abstract}

PACS numbers: 95.30.Sf, 98.62.Sb, 97.60.Lf

Keywords: Relativity; Gravitation; Tidal Charged black hole; Gauss-Bonnet; Effect of Plasma; Shadow

\section{INTRODUCTION}

Einstein's theory of general relativity (GR) is a wonderful gravity's theory and was borned in 1916, Einstein intelligently anticipated the presence of gravitational waves and gravitational lensing in his theory of (GR) [1]. Black Holes (BHs) are very interesting and captivating substance in the universe. It is assumed that various types of BHs are living in the universe but it is not confirmed experimentally till now. The black hole physics is very important because it plays a vital role in discovery of gravitational wave [2] and further BHs physics is used for more crucial level like entropy and the information paradox [3]. It also used in an interesting aspects of gravitational lensing. As indicated by Einstein, when light experiences an array of huge masses in its way towards the viewer then the light bends from the array and form the gravitational lens. When light passing from the array of galaxies then they divert the light's path by using gravitational fields and in the background they generating disturbance for the origin. Strong lensing generates curves and rings, for example, the Einstein's ring, while weak lensing is a consequence of small bends with amplifications too little to even think about detecting, except if found the middle value of over various systems.

The gravitational lensing is a useful system to comprehend the galaxies, dark matter of the universe, dark energy and the universe [4]. As the main gravitational lensing perception by the Eddington, a huge work on gravitational lensing have been accomplished for black holes, wormholes, cosmic strings and different items ([5]-[21]). Geodesic technique ([22]-[27]) was considered the main part for investigating the gravitational lensing and then in 2008, Gibbons and Werner changed the circumstances by introducing the Gauss-Bonnet theorem (GBT) [28]. With the help of GBT, they find the light's weak gravitational deflection in the static and spherically symmetric (SSS) spacetime e.g. Schwarzschild spacetime. In GBT the light's deflection angle can be determined by integrating Gaussian curvature of related optical metric. In GBT we can utilize a space $D_{R}$, which is limited by the photon beam just as a circular boundary curve $C_{R}$ which is situated at focus on the focal point here the photon beam meets the source and observer. It is expected that both origin and observer are at the coordinate distance R from the focal point. The GBT is communicated as pursues [28]:

$$
\iint_{D_{R}} \mathcal{K} d S+\oint_{\partial D_{R}} \kappa d t+\Sigma_{i} \theta_{i}=2 \pi \mathcal{X}\left(D_{R}\right)
$$

Here optical Gaussian curvature is denoted by $\mathcal{K}$ and an areal component is denoted by dS. Subsequently thinking about the Euler characteristic. $\mathcal{X}\left(D_{R}\right)=1$, also added in the jump angles $\Sigma_{i} \theta_{i}=\pi$, the deflection angle is acquired

\footnotetext{
*Electronic address: wajiha.javed@ue.edu.pk; wajihajaved84@yahoo.com

†Electronic address: alihamza.ahg@gmail.com

‡Electronic address: ali.ovgun@emu.edu.tr; URL: https : / / a ovgun.weebly.com
} 
by utilizing the adopting condition doing in consistence with the straight line estimate [28]:

$$
\alpha=-\int_{0}^{\pi} \int_{\frac{b}{r \sin \phi}}^{\infty} \mathcal{K} d S .
$$

Note that deflection angle is denoted by $\alpha$. Then some other physicists study the light's deflection angle using the GBT. For example, the deflection angle of light studied for BHs and wormholes by the following writers ([29]-[42]), Ovgun et al. studied for different spacetimes like Schwarzschild-like spacetime in bumblebee gravity model ([43][49]) and Javed et al. studied the impact of various matter fields ([50]-[54]). Next, Ishihara et al. [55] expressed that it is conceivable to calculate the finite-distances's deflection angle (enormous effect parameter). As of late, Crisnejo and Gallo have contemplated the light's deflection angle in the presence of plasma medium [56].

Most of the scientist said that a supermassive BH exist at the center of or galaxy and they hope that a far away viewer can see the supermassive BH as a dark space in the sky and it is called shadow of BH ([57]-[69]). Some say that which we called shadow, it is just the image of a event horizon and its size depend on the angle between the viewer and the event horizon according to Euclidean geometry. As a matter of fact, the shadow's boundary compares to light beams that asymptotically access the photon circle and not the skyline. In addition, photon beams don't move straight in Euclidean geometry yet they are bowed. Due to these reasons, the precise distance across of the shadow is really greater than the innocent Euclidean gauge recommends. For the dark gap at the focal point of our cosmic system, it adds up to around 53 ìas though the Euclidean gauge gives just around 20 ìas. At present, two ventures are in progress to watch the shadow that would give significant data on the minimal item at the focal point of our universe. These activities, which are going to utilize (sub)millimeter VLBI perceptions with radio telescopes appropriated over the Earth, are the Event Horizon Telescope and the Black Hole Cam [57].

The paper is organized as follows: in Section II we derive the optical metric and find the deflection angle in Section III. In Section IV we discus the graphical behavior and find the angle in the presence of plasma medium in Section V. Next, we find the graphical behavior in the presence of plasma in Section VI and calculate null geodesic in Section VII. Further we calculate shadow in Section VIII and also calculate shadow in the presence of plasma in Section IX and finally we conclude our results in Section X.

\section{OPTICAL METRIC OF TCBH}

The line element of a static, spherically symmetric TCBH is given by $[70,71]$

$$
d s^{2}=-B(r) d t^{2}+\frac{d r^{2}}{B(r)}+r^{2} d \Omega_{2}^{2},
$$

here

$$
B(r)=1-\frac{2 M}{M_{p}^{2} r}+\frac{q}{M_{5}^{2} r^{2}},
$$

and

$$
d \Omega_{2}^{2}=d \theta^{2}+\sin ^{2} \theta d \phi^{2}
$$

here $\mathrm{BH}^{\prime}$ s mass is denoted by $M$ and the dimensionless tidal charge is denoted by $q$ and $M_{p}(=1.2 * 1016 T e v)$ denotes the effective Plank mass on the brane and $M_{5}$ denotes the fundamental Planck scale in the 5D bulk.

As both the origin and viewer lies in the same plane, so let $\left(\theta=\frac{\pi}{2}\right)$. For obtaining optical metric we take $d s^{2}=0$,

$$
d t^{2}=\frac{d r^{2}}{B^{2}(r)}+\frac{r^{2} d \phi^{2}}{B(r)} .
$$

Now with the help of Eq.(2), the non-zero Christofell symbols are defined as

$$
\Gamma_{00}^{0}=\frac{B^{\prime}(r)}{B(r)} \quad, \quad \Gamma_{01}^{1}=\frac{1}{r}-\frac{B^{\prime}(r)}{2 B(r)}
$$

and

$$
\Gamma_{11}^{0}=-r B+\frac{r^{2} B^{\prime}(r)}{2}
$$


and Ricci Scalar for optical metric is obtained as:

$$
\mathcal{R}=B^{\prime \prime}(r) B(r)-\frac{\left(B^{\prime}(r)\right)^{2}}{2} .
$$

Then we calculate Gaussian optical metric as:

$$
\mathcal{K}=\frac{\text { RicciScalar }}{2}
$$

After doing some simple calculation, Gaussian optical curvature is written as:

$$
\mathcal{K} \approx-\frac{-2 M}{r^{3} M_{p}^{2}}-\frac{6 q M}{r^{5} M_{p}^{2} M_{5}^{2}}+\frac{3 q}{r^{4} M_{5}^{2}}+\mathcal{O}\left(M^{2}, q^{2}\right) .
$$

\section{DEFLECTION ANGLE OF TCBH}

In this section we find the deflection angle by TCBH with the help of GBT. We apply the GBT on this area $\mathcal{E}_{S}$, given as [28]

$$
\iint_{\mathcal{F}_{T}} \mathcal{K} d S+\oint_{\partial \mathcal{F}_{T}} k d t+\sum_{l} \epsilon_{l}=2 \pi \mathcal{Z}\left(\mathcal{F}_{T}\right)
$$

here $\mathcal{K}$ denote the Gaussian curvature and $k$ denote the geodesic curvature as; $k=\bar{g}\left(\nabla_{\dot{\beta}} \dot{\beta}, \ddot{\beta}\right)$ so, $\bar{g}(\dot{\beta}, \dot{\beta})=1, \ddot{\beta}$ shows unit acceleration vector and the $\epsilon_{l}$ shows the exterior angle at the lth vertex. Since $T \rightarrow \infty$, both of the jump angles curtail to $\pi / 2$ and then we have $\theta_{O}+\theta_{T} \rightarrow \pi$. Euler characteristic is $\mathcal{Z}\left(\mathcal{F}_{T}\right)=1$, as $\mathcal{F}_{T}$ is non singular. So,

$$
\iint_{\mathcal{F}_{T}} \mathcal{K} d S+\oint_{\partial \mathcal{F}_{T}} k d t+\epsilon_{l}=2 \pi \mathcal{Z}\left(\mathcal{F}_{T}\right)
$$

here, $\epsilon_{l}=\pi$ demonstrates that both $\alpha_{\bar{g}}$ and the total jump angle is a geodesic and $\mathcal{Z}$ is the Euler characteristic number and it is equal to 1 . As $T \rightarrow \infty$, then we have $k\left(E_{T}\right)=\mid \nabla_{\dot{E}_{T}} \dot{E}_{S T \mid}$. Since, radial component of geodesic curvature is written as [28]

$$
\left(\nabla_{\dot{E}_{T}} \dot{E}_{T}\right)^{r}=\dot{E}_{T}^{\phi} \partial_{\phi} \dot{E}_{T}^{r}+\Gamma_{11}^{0}\left(\dot{E}_{T}^{\phi}\right)^{2}
$$

For large $T, E_{T}:=r(\phi)=T=$ const. Hence, the equation Eq.(7) becomes $\left(\dot{E}_{T}^{\phi}\right)^{2}=\frac{A^{2}(r) B(r)}{r^{2}}$. As $\Gamma_{11}^{0}=-r B+\frac{r^{2} B^{\prime}(r)}{2}$, so it becomes

$$
\left(\nabla_{\dot{E}_{T}^{r}} \dot{E}_{T}^{r}\right)^{r} \rightarrow \frac{-1}{T}
$$

As geodesic curvature is free from topological defects so, $k\left(E_{T}\right) \rightarrow T^{-1}$. By using the optical metric Eq.(4) and written as $d t=T d \phi$. Hence, we have;

$$
k\left(E_{T}\right) d t=\frac{1}{T} T d \phi
$$

By combining all the result we have,

$$
\iint_{\mathcal{F}_{T}} \mathcal{K} d s+\oint_{\partial \mathcal{F}_{T}} k d t={ }^{T \rightarrow \infty} \iint_{U_{\infty}} \mathcal{K} d S+\int_{0}^{\pi+\Theta} d \phi .
$$

Light ray in week field limit at zeroth order is written as $r(t)=b / \sin \phi$. Using (5) and (11), the deflection angle is written as [28]

$$
\Theta=-\int_{0}^{\pi} \int_{b / \sin \phi}^{\infty} \mathcal{K} \sqrt{\operatorname{det} \bar{g}} d r d \phi
$$

here $\sqrt{\operatorname{det} \bar{g}} \approx r d r$.

After replacing the main terms of Gaussian curvature Eq.(4) into Eq.(11), the deflection angle is obtained as:

$$
\Theta \approx \frac{4 M}{b M_{p}^{2}}-\frac{3 q \pi}{4 b^{2} M_{5}^{2}}+\mathcal{O}\left(M^{2}, q^{2}\right)
$$




\section{GRAPHICAL ANALYSIS FOR NON-PLASMA MEDIUM}

In this section we obtained the graphical behavior of TCBH's deflection angle. We checked the graphical behavior in correspondence of deflection angle $\Theta$ with impact parameter $b$ by varying the value of dimensionless tidal charge $q$. In these graphs we take $M_{p}$ and $M_{5}$ are equal to 1.

\section{A. Deflection angle $\Theta$ w.r.t Impact parameter $b$}
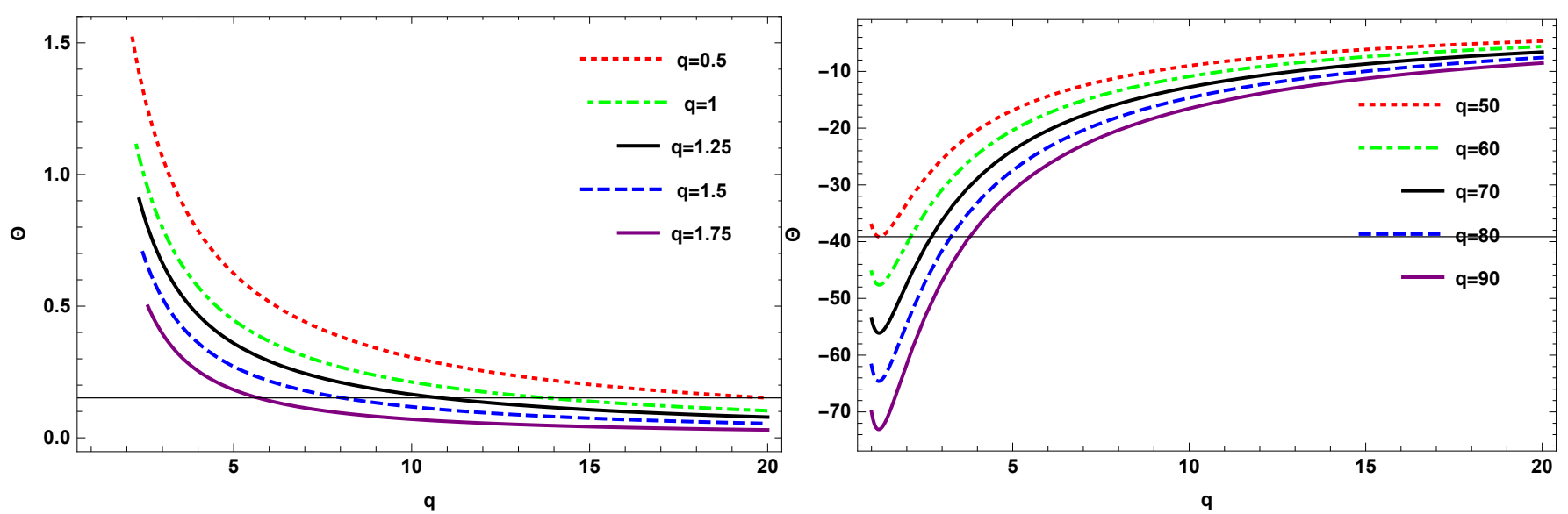

Figure 1: Relation between $\Theta$ and $b$.

- Figure 1 demonstrates the relation of $\Theta$ w.r.t $b$ for different values of $q$.

1. In left plot, by considering the value of $q=0.5$, deflection angle going to increase gradually and by increasing the values of $q$ 's the deflection angle decreases manually from graph of $q=0.5$ and then going to increase toward positive side.

2. In right figure, graphical behavior shows the negative behavior and buy increasing the values of $q$, deflection angle also decrease toward negative side.

\section{EFFECT OF PLASMA ON GRAVITATIONAL LENSING}

In this portion we check that how a plasma medium effect the gravitational lensing of TCBH. Now, we assume the $\mathrm{TCBH}$ in the presence of plasma illustrated by the refractive index $n,[56]$

$$
n^{2}(r, \omega(r))=1-\frac{\omega_{e}^{2}(r)}{\omega_{\infty}^{2}(r)} .
$$

Here, refractive index is defined as in this case;

$$
n(r)=\sqrt{1-\frac{\omega_{e}^{2}}{\omega_{\infty}^{2}}\left(1-\frac{2 M}{M_{p}^{2} r}+\frac{q}{M_{5}^{2} r^{2}}\right) .}
$$

and the line element is written as

$$
d s^{2}=-B(r) d t^{2}+\frac{1}{B(r)} d r^{2}+r^{2} d \Omega_{2}^{2}
$$

and

$$
B(r)=1-\frac{2 M}{M_{p}^{2} r}+\frac{q}{M_{5}^{2} r^{2}} .
$$

as both the origin and viewer are in the same plane. So, put $\left(\theta=\frac{\pi}{2}\right)$. For getting optical metric we let $d s^{2}=0$, as[56]

$$
d t^{2}=g_{l m}^{o p t} d x^{l} d x^{m}=n^{2}\left[\frac{d r^{2}}{B^{2}(r)}+\frac{r^{2} d \phi^{2}}{B(r)}\right]
$$


with determinant $g_{l m}^{o p t}$,

$$
\sqrt{g^{o p t}}=r\left(1-\frac{\omega_{e}^{2}}{\omega_{\infty}^{2}}\right)+\frac{M}{M_{p}^{2}(r)}\left(3+\frac{\omega_{e}^{2}}{\omega_{\infty}^{2}}\right)-\frac{q}{2 M_{5}^{2} r}\left(3+\frac{\omega_{e}^{2}}{\omega_{\infty}^{2}}\right) .
$$

By using Eq.(16),we can calculate the non-zero Christofell symbols as;

$$
\begin{gathered}
\Gamma_{00}^{0}=\left(1+\frac{\omega_{e}^{2} B}{\omega_{\infty}^{2}}\right)\left[-B^{\prime} B^{-1}\left(1-\frac{\omega_{e}^{2} B}{\omega_{\infty}^{2}}\right)-\frac{B^{\prime} \omega_{e}^{2}}{2 \omega_{\infty}^{2}}\right], \\
\Gamma_{10}^{1}=\left(1+\frac{\omega_{e}^{2} B}{\omega_{\infty}^{2}}\right)\left[r^{-1}\left(1-\frac{\omega_{e}^{2} B}{\omega_{\infty}^{2}}-\frac{B^{\prime} B^{-1}}{2}\left(1-\frac{\omega_{e}^{2} B}{\omega_{\infty}^{2}}\right)-\frac{B^{\prime} \omega_{e}^{2}}{2 \omega_{\infty}^{2}}\right]\right.
\end{gathered}
$$

and

$$
\Gamma_{11}^{0}=\left(1+\frac{B \omega_{e}^{2}}{\omega_{\infty}^{2}}\right)\left[-r B\left(1-\frac{B \omega_{e}^{2}}{\omega_{\infty}^{2}}\right)+\frac{r^{2} B^{\prime}}{2}\left(1-\frac{B \omega_{e}^{2}}{\omega_{\infty}^{2}}\right)+\frac{r^{2} B}{2} \frac{B^{\prime} \omega_{e}^{2}}{\omega_{\infty}^{2}}\right]
$$

In terms of curvature tensor, the Gaussian curvature can be calculated in this way

$$
\mathcal{K}=-3 \frac{M \omega_{e}^{2}}{r^{3} \omega_{\infty}^{2} M_{p}^{2}}-2 \frac{M}{M_{p}^{2} r^{3}}+5 \frac{q \omega_{e}^{2}}{\omega_{\infty}^{2} M_{5}^{2} r^{4}}+3 \frac{q}{M_{5}^{2} r^{4}}-26 \frac{q M \omega_{e}^{2}}{\omega_{\infty}^{2} M_{5}^{2} M_{p}^{2} r^{5}}-6 \frac{q M}{M_{5}^{2} M_{p}^{2} r^{5}}
$$

With the help of GBT we obtained the deflection angle of TCBH in the presence of plasma medium and we relate it with non-plasma. So, for obtaining deflection angle in weak field limit, we apply the condition of $r=\frac{b}{\sin \phi}$ at 0 th order.

$$
\Theta=-\lim _{R \rightarrow 0} \int_{0}^{\pi} \int_{\frac{b}{\sin \phi}}^{R} \mathcal{K} d S
$$

using this Eq.(10), the deflection angle of light in the presence of plasma medium is written as;

$$
\Theta=-6 \frac{M \omega_{e}^{2}}{b \omega_{\infty}^{2} M_{p}^{2}}-4 \frac{M}{b M_{p}{ }^{2}}+5 / 4 \frac{q \omega_{e}^{2} \pi}{b^{2} \omega_{\infty}{ }^{2} M_{5}{ }^{2}}+3 / 4 \frac{q \pi}{b^{2} M_{5}{ }^{2}}+\mathcal{O}\left(M^{2}, q^{2}, \frac{\omega_{e}^{3}}{\omega_{\infty}^{3}}\right) .
$$

\section{GRAPHICAL ANALYSIS FOR PLASMA MEDIUM}

In this portion we check that how TCBH's deflection angle behave in the presence of plasma medium. Here, we take $\frac{\omega_{e}}{\omega_{\infty}}=10^{-1}$ and observe the deflection angle by changing the value of dimensionless tidal charge $q$. We also take $M_{p}$ and $M_{5}$ are equal to 1.

\section{A. Deflection angle w.r.t Impact parameter}
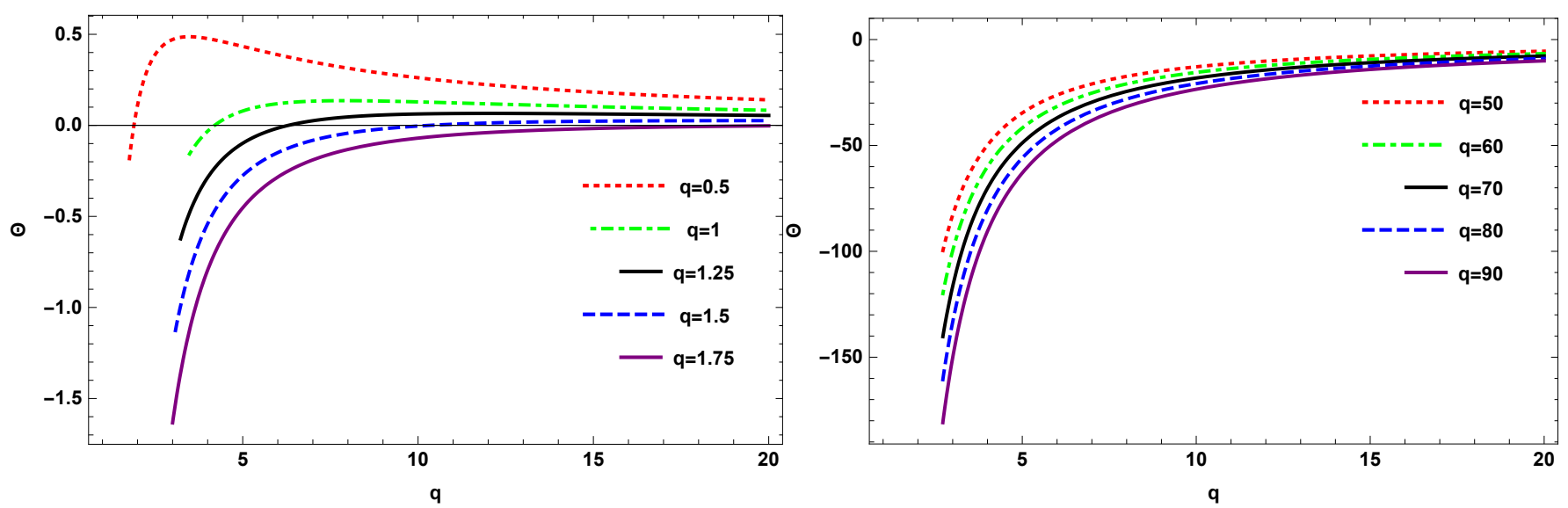

Figure 2: Relation between $\Theta$ and $b$. 
- Figure 2 demonstrates the relation of $\Theta$ with $b$ for different values of $b$.

1. In 1st plot we observed that for value of $q=0.5$, the deflection angle first increase towards positive and then suddenly decrease toward negative side at some point. For values of $q$ grater than 0.5 , the deflection angle gradually decrease from positive to negative side and goes on toward the negative side.

2. In 2nd plot we observed that for large values of $q$ the deflection angle goes towards the negative side and by increasing the values of $q$, it continuously move toward negative side.

\section{NULL GEODESIC IN A TCBH}

The Lagrangian representing the motion of light in the TCBH's spacetime using this Eq.(1) is written as;

$$
2 \mathcal{L}=-\left(1-\frac{2 M}{M_{p}^{2} r}+\frac{q}{M_{5}^{2} r^{2}}\right) \dot{t}^{2}+\left(1-\frac{2 M}{M_{p}^{2} r}+\frac{q}{M_{5}^{2} r^{2}}\right) \dot{r}^{2}+r^{2} \dot{\theta}^{2}+r^{2} \sin ^{2} \theta \dot{\phi}^{2},
$$

here derivative w.r.t affine parameter $\lambda$ is represented by an overdot. As Lagrangian is not depend on $\mathrm{t}$ and $\phi$ so we introduce two new constants named as energy $E$ and the angular momentum $L$. Where the values of these constants is defined as;

$$
p_{t}=\frac{\partial L}{\partial \dot{t}}=-\left(1-\frac{2 M}{M_{p}^{2} r}+\frac{q}{M_{5}^{2} r^{2}}\right) \dot{t}=-E
$$

and

$$
p_{\phi}=\frac{\partial L}{\partial \dot{\phi}}=r^{2} \sin ^{2} \theta \dot{\phi}=L
$$

For finding the restrictions of geodesic, we use these new introduced constants as;

$$
\frac{d t}{d \lambda}=\dot{t}=\frac{E}{1-\frac{2 M}{M_{p}^{2} r}+\frac{q}{M_{5}^{2} r^{2}}} \quad, \quad \frac{d \phi}{d \lambda}=\dot{\phi}=\frac{L}{r^{2} \sin ^{2} \theta} .
$$

Now, we define the new parts of momentum named as $r$-part and $\theta$-par;

$$
p_{r}=\frac{\partial L}{\partial \dot{r}}=\frac{\dot{r}}{1-\frac{2 M}{M_{p}^{2} r}+\frac{q}{M_{5}^{2} r^{2}}} \text { and } p_{\theta}=\frac{\partial L}{\partial \dot{\theta}}=r^{2} \dot{\theta}
$$

With the help of Hamilton-Jacobi equation, we find the values of $r$-part and $\theta$-part of the geodesic equation as;

$$
\frac{\partial S}{\partial \lambda}=-\frac{1}{2} g^{\mu \nu} \frac{\partial S}{\partial x^{\mu}} \frac{\partial S}{\partial x^{\nu}},
$$

and for photons $\left(m_{0}=0\right)$, Eq. (24) can give us result of the following type,

$$
S=-E t+L \phi+S_{r}(r)+S_{\theta}(\theta)
$$

where $S_{r}$ depends on $r$ and $S_{\theta}$ depends on $\theta$. Now we get the Carter constant $( \pm \mathcal{K})$ [66] by separating the values of $r$ and $\theta$ and we get these values of $r$ and $\theta$ by replacing Eq. (25) into Eq. (24) and also substituting the values of contravariant metric, i.e., $g^{\mu \nu}$, we have

$$
\begin{aligned}
\frac{1}{\sqrt{1-\frac{2 M}{M_{p}^{2} r}+\frac{q}{M_{5}^{2} r^{2}}} \frac{d r}{d \lambda}}={ }_{-}^{+} \sqrt{R}(r), \\
r^{2} \frac{d \theta}{d \lambda}={ }_{-}^{+} \sqrt{T}(\theta),
\end{aligned}
$$

here the values of $R$ and $\theta$ can be defined as,

$$
\begin{array}{r}
R(r)=\frac{E^{2}}{1-\frac{2 M}{M_{p}^{2} r}+\frac{q}{M_{5}^{2} r^{2}}}-\frac{\mathcal{K}}{r^{2}}, \\
T(\theta)=\mathcal{K}-\frac{L^{2}}{\sin ^{2} \theta} .
\end{array}
$$


Now, Equation $S_{r}$ can be written as

$$
\frac{d r^{2}}{d \lambda}+V_{e f f}=0
$$

with

$$
V_{e f f}=-\left(1-\frac{2 M}{M_{p}^{2} r}+\frac{q}{M_{5}^{2} r^{2}}\right) R(r) .
$$

We see that effective potential depend on $\mathrm{BH}^{\prime}$ s mass denoted by $M$ and the dimensionless tidal charge denoted by $q$ and effective Plank mass on the brane denoted by $M_{p}$ and fundamental Planck scale in the 5D bulk denoted by $M_{5}$ and radius $r$ and $R(r)$. Now we change these parameters to new impact parameters such as $\xi=\frac{L}{E}$ and $\eta=\frac{\mathcal{K}}{E^{2}}$. Now we change the value of $\mathcal{R}$ with respect to these new impact parameters.

$$
R=E^{2}\left[\frac{1}{1-\frac{2 M}{M_{p}^{2} r}+\frac{q}{M_{5}^{2} r^{2}}}-\frac{\eta}{r^{2}}\right]
$$

\section{SHADOW OF TCBH}

Here, in this section we find the shadow of TCBH and we discussed in detail about shadow in the introduction. Now for finding the shadow, we find the unstable circular photons orbits. For this we must satisfy this,

$$
R=0 \quad \text { and } \quad R^{\prime}=0
$$

where prime ( $/$ ) means differentiation w.r.t $r$. Putting (30) into (31), we obtained

$$
\eta+\xi^{2}=\frac{r^{2}}{B(r)}
$$

and

$$
\frac{B^{\prime}(r)}{B(r)}=\frac{2}{r}
$$

the photon sphere radius $r$ is found as follows:

$$
\eta=\frac{r^{2}}{1-\frac{2 M}{M_{p}^{2} r}+\frac{q}{M_{5}^{2} r^{2}}}, \quad r=\frac{3 M}{2 M_{p}^{2}}+\frac{\sqrt{\frac{9 M^{2}}{M_{p}^{2}}-\frac{8 q}{M_{5}^{2}}}}{2} .
$$

Where impact parameters depends on BH's mass denoted by $M$ and the dimensionless tidal charge denoted by $q$ and effective Plank mass on the brane denoted by $M_{p}$ and fundamental Planck scale in the 5D bulk denoted by $M_{5}$ and radius $r$. So Eq. (34) give detail about the boundary of the shadow and an observer which is far away from the $\mathrm{BH}$ can find this shadow in this sky and we make new coordinates in the observer's sky named as the celestial coordinates $(\alpha, \beta)$, and we relate these coordinates with impact parameters $(\xi, \eta)$. These coordinates are defines in these papers ([67],[68]) as;

$$
\begin{array}{r}
\alpha=\lim _{r_{0} \rightarrow \infty}\left(r_{0}^{2} \sin \theta_{0}\right) \frac{d \phi}{d r}, \\
\beta=\lim _{r_{0} \rightarrow \infty} r_{0}^{2} \frac{d \theta}{d r},
\end{array}
$$

Where $r_{0}$ represents the distance between the viewer and the $\mathrm{BH}$ and $\theta_{0}$ denotes the angular coordinates of the observer called "inclination angle". After putting the equations of four-velocities into Eq. (35), and doing some calculation, we obtain these celestial coordinates as,

$$
\alpha=-\frac{\xi}{\sin \theta_{0}} \quad \text { and } \quad \beta=\sqrt{\eta-\frac{\xi^{2}}{\sin \theta_{0}^{2}}} .
$$


With the help of these equations and using the impact parameters we now make the shape of TCBH's shadow and for plotting the shadows's shape we plot $\alpha$ versus $\beta$ which give detail about the boundary of the TCBH in the observer's sky and these plots are seen in the Fig 3.
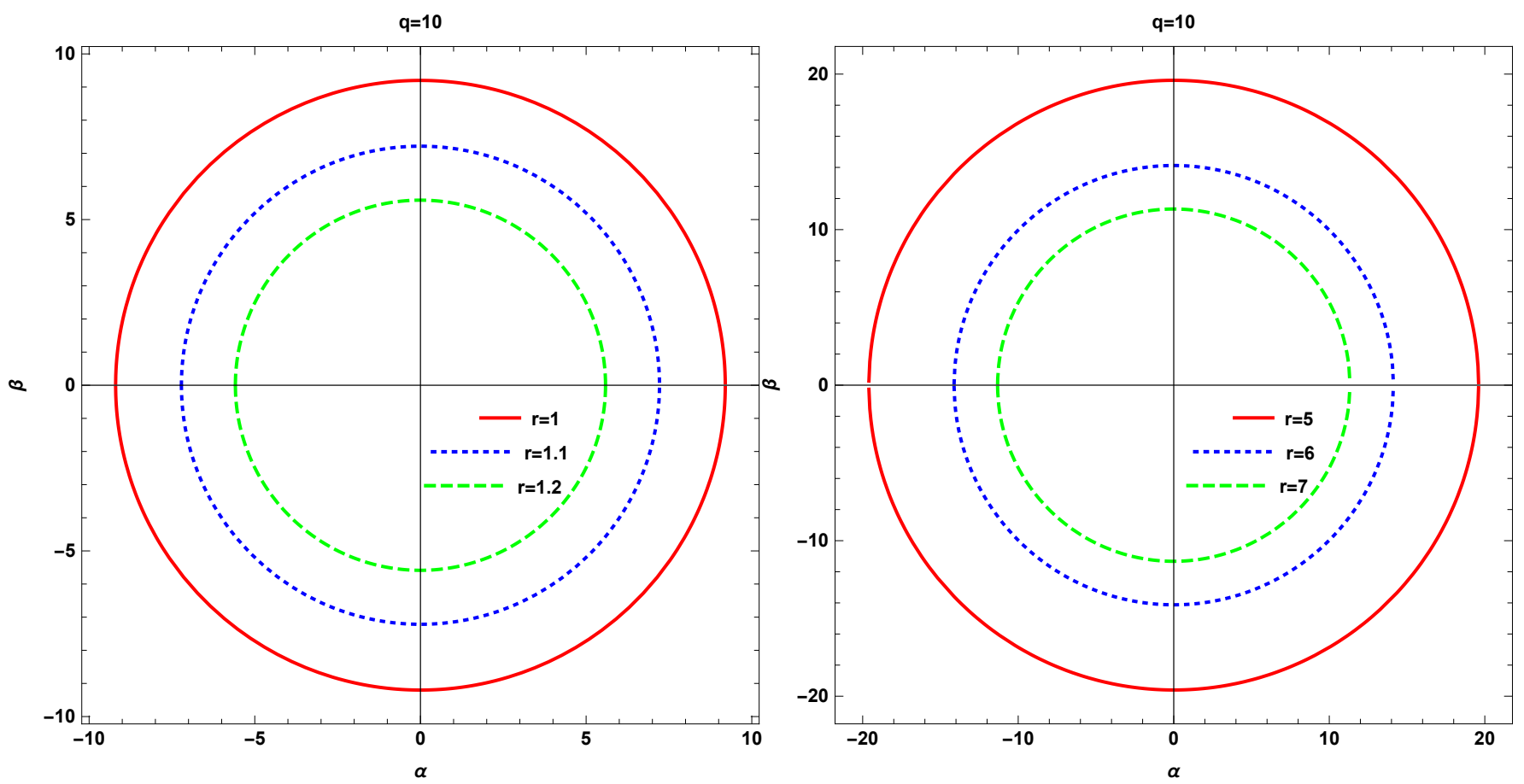

Figure 3: Graphs displaying the shadow of TCBH.

We plots the different graphs by changing the values of the dimensionless tidal charge $q$ and radial coordinate $r$, in these plots we discuss about the shadow for small and large values of $q$ and by varying the values of $r$ in the equatorial plan by putting $\theta_{0}=\frac{\pi}{2}$. We saw that shadow's shape is a perfect square and its shape as shown in Fig. 3 . In these graphs we see that for small values of $r$ and for large values of $r$ shadow shows the same behavior for fix vale of $q=10$ and shadow is decreasing by increasing the value of $r$.

\section{EFFECT OF PLASMA ON SHADOW OF TCBH}

In Paper [69] we discuss in detail about the formula of the calculating the formula of shadow of a spherically symmetric spacetime. The line element of spherically symmetric TCBH is defined in this Eq.(1) and we can write as

$$
d s^{2}=-A(r) d t^{2}+\frac{d r^{2}}{B(r)}+r^{2} d \Omega_{2}^{2}
$$

here

$$
A(r)=[B(r)]^{-1}=1-\frac{2 M}{M_{p}^{2} r}+\frac{q}{M_{5}^{2} r^{2}}, \quad \text { and } \quad D(r)=r^{2},
$$

and here we also check the TCBH's shadow in the presence of plasma medium. The refractive index $n$ can be written as;

$$
n^{2}(r, \omega(r))=1-\frac{\omega_{e}^{2}(r)}{\omega_{\infty}^{2}} .
$$

Here from reference[69], w can define the $h(r)$ as;

$$
h\left(r^{2}\right)=r^{2}\left(\frac{1}{1-\frac{2 M}{M_{p}^{2} r}+\frac{q}{M_{5}^{2} r^{2}}}-\frac{\omega_{e}^{2}(r)}{\omega_{\infty}^{2}}\right) .
$$


We can calculate the radius of a photon sphere by computing the above equation as,

$$
0=\frac{d}{d r}\left(h(r)^{2}\right)
$$

In this case the value of $h(r)$ is defined by (39) and we use circumstances (40) for a photon sphere becomes

$$
0=\frac{4 r^{3}\left(r^{2}-\frac{2 M r}{M_{p}^{2}}+\frac{q}{M_{5}^{2}}\right)-r^{4}}{\left(r^{2}-\frac{2 M r}{M_{p}^{2}}+\frac{q}{M_{5}^{2}}\right)^{2}}-2 r \frac{\omega_{e}^{2}(r)}{\omega_{\infty}^{2}}-2 r^{2} \frac{\omega_{e}(r) \omega_{e}\left(r^{\prime}\right)}{\omega_{\infty}^{2}} .
$$

Angular radius of the shadow is defined as,

$$
\sin ^{2} \alpha_{s h}=\frac{r_{p h}^{2}\left(\frac{1}{1-\frac{2 M}{M_{p}^{2} r_{p h}}+\frac{q}{M_{5}^{2} r_{p h}^{2}}}-\frac{\omega_{e}^{2}\left(r_{p h}\right)}{\left.\omega_{\infty}^{2}\right)}\right.}{r_{p h}^{2}\left(\frac{1}{1-\frac{2 M}{M_{p}^{2} r_{p h}}+\frac{q}{M_{5}^{2} r_{p h}^{2}}}-\frac{\omega_{e}^{2}\left(r_{p h}\right)}{\omega_{\infty}^{2}}\right)}
$$

where $r_{p h}$ has to be determined from (42). For vacuum, $\omega_{e}(r)=0$, our consideration gives

$$
h\left(r^{2}\right)=r^{2}\left(\frac{1}{1-\frac{2 M}{M_{p}^{2} r}+\frac{q}{M_{5}^{2} r^{2}}}\right) .
$$

and

$$
\sin ^{2} \alpha_{s h}=\frac{r_{p h}^{2}\left(\frac{1}{1-\frac{2 M}{M_{p}^{2} r_{p h}}+\frac{q}{M_{5}^{2} r_{p h}^{2}}}\right)}{r_{p h}^{2}\left(\frac{1}{1-\frac{2 M}{M_{p}^{2} r_{p h}}+\frac{q}{M_{5}^{2} r_{p h}^{2}}}\right)}
$$

where the positive value of $r_{p h}$ can be calculated as;

$$
r_{p h}=\frac{1+\frac{2 M}{M_{p}^{2}}+\sqrt{\left(1+\frac{2 M}{M_{p}^{2}}\right)^{2}-\frac{4 q}{M_{5}^{2}}}}{2}
$$

\section{CONCLUSION}

In this paper, we first of all find the deflection angle of TCBH with the help of Gaussian curvature. We find the angle by a famous theorem named as GBT proposed by Gibbons and Werner. The deflection angle is described as

$$
\Theta \approx \frac{4 M}{b M_{p}^{2}}-\frac{3 q \pi}{4 b^{2} M_{5}^{2}}+\mathcal{O}\left(M^{2}, q^{2}\right)
$$

This shows that angle depends on BH's mass denoted by $M$ and the dimensionless tidal charge denoted by $q$ and effective Plank mass on the brane denoted by $M_{p}$ and fundamental Planck scale in the 5D bulk denoted by $M_{5}$ and impact parameter $b$. After calculating the deflection angle of TCBH we check its graphical behavior by varying the values of $q$ and by fixing all the other constants. In the next step we move toward the deflection angle of TCBH in the presence of plasma medium. We find this angle also by the same method named as GBT. This angle in the presence of plasma is defined below

$$
\Theta=-6 \frac{M \omega_{e}^{2}}{b \omega_{\infty}^{2} M_{p}^{2}}-4 \frac{M}{b M_{p}^{2}}+5 / 4 \frac{q \omega_{e}^{2} \pi}{b^{2} \omega_{\infty}^{2} M_{5}^{2}}+3 / 4 \frac{q \pi}{b^{2} M_{5}{ }^{2}}+\mathcal{O}\left(M^{2}, q^{2}, \frac{\omega_{e}^{3}}{\omega_{\infty}^{3}}\right) .
$$

After neglecting the plasma medium effect we find the same angle as we find in the non-plasma case. Now if we neglect the plasma effect $\left(\frac{\omega_{e}}{\omega_{\infty}} \rightarrow 0\right)$ then this deflection angle Eq. (20) reduces into this angle Eq. (46). this shows 
the correctness of our angle in the presence of plasma medium. After calculating the effect of plasma, we find the graphical behavior of TCBH in the presence of plasma medium. It does not shows the same behavior as the behavior without plasma.

Now in this paper we also find the shadow of TCBH, for this we first of all find the Null geodesic of TCBH and then convert it to new impact parameters named as energy $E$ and the angular momentum $L$. After that we calculate the shadow and shows his image in the far away observer's sky, for this we convert our results into celestial coordinates $(\alpha, \beta)$. After calculating the shadow, we plots the graphs of the shadow and discuss it by varying values of radial coordinate $r$. We also discuss the shadow of TCBH in the presence of plasma medium and discuss its effect on shadow of TCBH.

\section{Acknowledgments}

This work was supported by Comisión Nacional de Ciencias y Tecnología of Chile through FONDECYT Grant $N^{\circ}$ 3170035 (A. Ö.).

[1] A. Einstein, Science 84, 506 (1936)

[2] B. P. Abbott et al. [LIGO Scientific and Virgo Collaborations], Phys. Rev. Lett. 116, no. 6, 061102 (2016).

[3] S. D. Mathur, Class. Quant. Grav. 26, 224001 (2009).

[4] M. Bartelmann, Class. Quant. Grav. 27, 233001 (2010).

[5] C. R. Keeton, C. S. Kochanek and E. E. Falco, Astrophys. J. 509, 561 (1998).

[6] A. Bhadra, Phys. Rev. D 67, 103009 (2003).

[7] R. Whisker, Phys. Rev. D 71, 064004 (2005).

[8] S. b. Chen and J. l. Jing, Phys. Rev. D 80, 024036 (2009).

[9] K. K. Nandi, Y. Z. Zhang and A. V. Zakharov, Phys. Rev. D 74, 024020 (2006).

[10] E. F. Eiroa, G. E. Romero and D. F. Torres, Phys. Rev. D 66, 024010 (2002).

[11] S. Mao and B. Paczynski, Astrophys. J. 374, L37 (1991).

[12] V. Bozza, Phys. Rev. D 66, 103001 (2002).

[13] H. Hoekstra, H. K. C. Yee and M. D. Gladders, Astrophys. J. 606, 67 (2004).

[14] K. S. Virbhadra and G. F. R. Ellis, Phys. Rev. D 65, 103004 (2002).

[15] O. Gurtug and M. Mangut, Phys. Rev. D 99, no. 8, 084003 (2019)

[16] K. S. Virbhadra and G. F. R. Ellis, Phys. Rev. D 62, 084003 (2000).

[17] O. Kasikci and C. Deliduman, Phys. Rev. D 100, no. 2, 024019 (2019)

[18] E. Gallo and O. M. Moreschi, Phys. Rev. D 83, 083007 (2011).

[19] G. Crisnejo and E. Gallo, Phys. Rev. D 97, no. 8, 084010 (2018).

[20] M. Sharif and S. Iftikhar, Astrophys. Space Sci. 357, no. 1, 85 (2015).

[21] G. W. Gibbons, Phys. Lett. B 308, 237 (1993).

[22] S. Weinberg, Gravitation and Cosmology: Principles and Applications of the General Theory of Relativity (Wiley, New York, 1972).

[23] A. Edery and M. B. Paranjape, Phys. Rev. D 58, 024011 (1998).

[24] J. Bodenner and C. Will, Am. J. Phys. 71, 770 (2003).

[25] K. Nakajima and H. Asada, Phys. Rev. D 85, 107501 (2012).

[26] W. G. Cao and Y. Xie, Eur. Phys. J. C 78, 191 (2018).

[27] C. Y.Wang, Y. F. Shen and Y. Xie, J. Cosmol. Astropart. Phys. 04 (2019) 022.

[28] G. W. Gibbons and M. C. Werner, Classical Quantum Gravity 25, 235009 (2008).

[29] K. Jusufi, I. Sakalli, and A. Övgün, Phys. Rev. D 96, 024040 (2017).

[30] Z. Li and A. Övgün, Phys. Rev. D 101, no. 2, 024040 (2020)

[31] K. Jusufi and A. Övgün, Phys. Rev. D 97, 024042 (2018).

[32] Y. Kumaran and A. Övgün, Chin. Phys. C 44, 025101 (2020).

[33] K. Jusufi, A. Övgün, J. Saavedra, Y. Vasquez, and P. A. Gonzalez, Phys. Rev. D 97, 124024 (2018).

[34] I. Sakalli, A. Ovgun, EPL 118(6), 60006 (2017).

[35] K. Jusufi and A. Övgün, Int. J. Geom. Meth. Mod. Phys. 16, no. 08, 1950116 (2019)

[36] K. Jusufi and A. Övgün, Phys. Rev. D 97, 064030 (2018).

[37] Z. Li, G. He and T. Zhou, Phys. Rev. D 101, no. 4, 044001 (2020).

[38] A. Övgün, G. Gyulchev, and K. Jusufi, Annals Phys. 406, 152 (2019).

[39] Z. Li and T. Zhou, Phys. Rev. D 101, no. 4, 044043 (2020)

[40] K. Jusufi, A. Övgün, A. Banerjee and I. Sakalli, Eur. Phys. J. Plus 134, no. 9, 428 (2019).

[41] Z. Li and J. Jia, Eur. Phys. J. C 80, no. 2, 157 (2020)

[42] K. Jusufi, M. C. Werner, A. Banerjee, and A. Övgün, Phys. Rev. D 95, 104012 (2017). 
[43] A. Övgün, K. Jusufi, and I. Sakalli, Ann. Phys. (Amsterdam) 399, 193 (2018).

[44] A. Övgün, K. Jusufi, and I. Sakalli, Phys. Rev. D 99, 024042 (2019).

[45] A. Övgün, I. Sakalli, and J. Saavedra, JCAP 1810, 041 (2018).

[46] A. Övgün, Universe 5, 115 (2019).

[47] A. Övgün, Phys. Rev. D 98, 044033 (2018).

[48] A. Övgün, I. Sakalli, and J. Saavedra, Annals Phys. 411, 167978 (2019).

[49] A. Övgün, Phys. Rev. D 99, 104075 (2019).

[50] W. Javed, R. Babar, and A. Övgün, Phys. Rev. D 99, 084012 (2019).

[51] W. Javed, R. Babar, and A. Övgün, Phys. Rev. D 100, 104032 (2019).

[52] W. Javed, j. Abbas and A. Övgün, Phys. Rev. D 100, no. 4, 044052 (2019).

[53] W. Javed, J. Abbas, A. Övgün, Eur. Phys. J. C 79, 694 (2019).

[54] W. Javed, M. Bilal Khadim, J. Abbas, A. Övgün, Preprints 2019, 2019120045 (doi: 10.20944/preprints201912.0045.v1).

[55] A. Ishihara, Y. Suzuki, T.Ono,T. Kitamura, H.Asada, Phys. Rev. D 94(8), 084015 (2016)

[56] G. Crisnejo, E. Gallo, Phys. Rev. D 97(12), 124016 (2018)

[57] K. Akiyama et al. [Event Horizon Telescope Collaboration], Astrophys. J. 875, no. 1, L1 (2019)

[58] R. A. Konoplya, T. Pappas and A. Zhidenko, Phys. Rev. D 101, no. 4, 044054 (2020)

[59] R. A. Konoplya, Phys. Lett. B 795, 1 (2019)

[60] X. Lu and Y. Xie, Eur. Phys. J. C 79, no. 12, 1016 (2019).

[61] A. Allahyari, M. Khodadi, S. Vagnozzi and D. F. Mota, JCAP 2002, no. 02, 003 (2020)

[62] R. Narayan, M. D. Johnson and C. F. Gammie, Astrophys. J. 885, no. 2, L33 (2019)

[63] C. Ding, C. Liu, R. Casana and A. Cavalcante, Eur. Phys. J. C 80, no. 3, 178 (2020)

[64] R. Shaikh and P. S. Joshi, JCAP 1910, no. 10, 064 (2019)

[65] I. Banerjee, S. Chakraborty and S. SenGupta, Phys. Rev. D 101, no. 4, 041301 (2020)

[66] B. Carter, Phys. Rev. 174, 1559 (1968).

[67] J.M. Bardeen, in Black holes, in Proceeding of the Les Houches Summer School, Session 215239, edited by C. De Witt and B.S. De Witt and B.S. De Witt (Gordon and Breach, New York, 1973).

[68] S. Chandrasekhar, The Mathematical Theory of Black Holes (Oxford University Press, New York, 1992).

[69] V. Perlick, O. Y. Tsupko and G. S. Bisnovatyi-Kogan, Phys. Rev. D 92, no. 10, 104031 (2015).

[70] N. Dadhich, R. Maartens, P. Papadopoulos and V. Rezania, Phys. Lett. B 487, 1 (2000).

[71] P. Pradhan, Int. J. Geom. Meth. Mod. Phys. 15, no. 01, 1850011 (2017). 